\title{
Paracetamol use in pregnancy - caution over causal inference from available data
}

\author{
Sura Alwan, Elizabeth A. Conover, Lorrie Harris-Sagaribay, Steven H. Lamm, \\ Sharon V. Lavigne, Shari I. Lusskin, Sarah G. Obican, Alfred N. Romeo (1), \\ Anthony R. Scialli and Katherine L. Wisner(D)
}

The Consensus Statement by Bauer et al. ${ }^{1}$ (Bauer, A. Z. et al. Paracetamol use during pregnancy - a call for precautionary action. Nat. Rev. Endocrinol. 17, 757-766 (2021) ${ }^{1}$ ) has, not surprisingly, drawn considerable public attention. We agree with the authors' call for a focused research effort to investigate the reported associations from observational studies concerning prenatal use of paracetamol (also known as acetaminophen or $N$-acetyl- $p$-aminophenol (APAP)) and adverse reproductive and neurodevelopmental outcomes. However, we caution against an inference of causality that is based upon inadequate evidence. Here we provide a consensus counterstatement to the conclusion of that paper.

We reviewed the literature on APAP use in pregnancy and adverse outcomes. These studies are limited by serious methodological problems, including failure to account for confounding, and elements of bias that make interpretation of the data challenging. APAP is a common over-the-counter drug that more than $60 \%$ of individuals recall having used sometime during pregnancy ${ }^{2}$. Differential exposure and misclassification of the timing, dose and duration of use of APAP during pregnancy is likely, as well as confusion with other analgesic drugs, which could lead to over-estimation of the true effect. Residual confounding from shared co-morbidities and genetic and environmental factors with exposure and outcome can bias associations away from the null. Pregnant people use APAP for migraine or fever, both of which have been associated with adverse effects on fetal neurological development ${ }^{3,4}$. Furthermore, individuals who use APAP in pregnancy for longer durations have a higher prevalence of self-reported mental health conditions, including depression and anxiety, and are reported to have more prevalent use of antidepressant medications, compared with pregnant individuals who use APAP for shorter durations ${ }^{2}$. The potential for a shared underlying genetic susceptibility to neurodevelopmental and psychiatric conditions being passed from parent to child must also be considered ${ }^{5,6}$. An important problem in combining data from several observational studies is the considerable variability in selection and adjustment of the potential confounders, which intensifies interpretation challenges ${ }^{7}$. Finally, the quality and validity of neurodevelopmental outcome definitions are problematic ${ }^{8}$. Attempts to globally quantify neurodevelopmental outcome definitions for clinical interpretation can be highly misleading, given their wide phenotypic presentations.

Although the authors acknowledge the vast limitations of past studies on this subject, they do not consider the clinical consequences that could result from their premature precautionary statements. Currently, there exists vaccine hesitancy among a notable percentage of the population due to overinterpretation of flawed studies and unfounded associations. This vaccine hesitancy undoubtedly results in negative consequences for pregnant individuals and for public health. Similarly, there are many examples in which undertreatment of maternal illnesses due to medication hesitancy poses a far greater risk to the fetus and mother than does exposure to the medication $^{9,10}$. Until better data for systematic synthesis become available, we urge against recommending such precautionary measures for APAP use in pregnancy and against the dissemination of information based on inconclusive and insufficient evidence. At the same time, we strongly encourage that such questions related to the safety of APAP use in pregnancy should continue to be investigated. Our statement is supported by 50 scientists, clinicians, epidemiologists and teratology information specialists affiliated with the Organization of Teratology Information Specialists and/or other partner organizations (Supplementary Box 1).

There is a reply to this letter by Bauer, A. Z. et al. Nat. Rev. Endocrinol. https://doi.org/ 10.1038/s41574-021-00610-1 (2021).
Sura Alwan ${ }^{\boxplus}$, Elizabeth A. Conover ${ }^{2}$ Lorrie Harris-Sagaribay ${ }^{3}$, Steven H. Lamm4, Sharon V. Lavigne ${ }^{5}$, Shari I. Lusskin ${ }^{6}$, Sarah G. Obican ${ }^{7}$, Alfred N. Romeo (iD ${ }^{8}$, Anthony R. Scialli ${ }^{9}$ and Katherine L. Wisner (iD ${ }^{10}$

'Department of Environmental and Occupational Health Sciences, School of Public Health, University of Washington, Seattle, WA, USA

${ }^{2}$ Division of Clinical Genetics, Munroe Meyer Institute, University of Nebraska Medical Center, Omaha, $N E, U S A$.

${ }^{3}$ MotherToBaby North Carolina, Asheville, NC, USA ${ }^{4}$ Center for Epidemiology and Maternal-Child Health, Washington, DC, USA

${ }^{5}$ MotherToBaby Connecticut, Division of Medical Genetics, UConn Health, Farmington, CT, USA.

${ }^{6}$ Department of Psychiatry and Obstetrics and Gynecology, Icahn School of Medicine at Mount Sinai, New York, NY, USA

${ }^{7}$ Department of Obstetrics and Gynecology, Morsani College of Medicine, University of South Florida, Tampa, FL, USA.

${ }^{8}$ MotherToBaby Utah, Utah Department of Health, Salt Lake City, UT, USA.

${ }^{9}$ Reproductive Toxicology Center, A Non-Profit Foundation, Washington, DC, USA

${ }^{10}$ Department of Psychiatry and Behavioral Sciences, Northwestern University Feinberg School of Medicine Chicago, IL, USA.

凶e-mail:alwans@uw.edu https://doi.org/10.1038/s41574-021-00606-x

1. Bauer, A. Z. et al. Paracetamol use during pregnancy - a call for precautionary action. Nat. Rev. Endocrinol. 17, 757-766 (2021).

2. Bandoli, G., Palmsten, K. \& Chambers, C.

Acetaminophen use in pregnancy: examining prevalence, timing, and indication of use in a prospective birth cohort. Paediatr. Perinat. Epidemiol. 34, 237-246 (2020)

3. Antoun, S. et al. Fever during pregnancy as a risk factor for neurodevelopmental disorders: results from a systematic review and meta-analysis. Mol. Autism 12, 60 (2021).

4. Skajaa, N. et al. Pregnancy, birth, neonatal, and postnatal neurological outcomes after pregnancy with migraine. Headache 59, 869-879 (2019).

5. Martin, J., Taylor, M. J. \& Lichtenstein, P. Assessing the evidence for shared genetic risks across psychiatric disorders and traits. Psychol. Med. 48, 1759-1774 (2018).

6. Leppert, B et al. Association of maternal neurodevelopmental risk alleles with early-life exposures. JAMA Psychiatry 76, 834-842 (2019).

7. Mathur, M. B. \& VanderWeele, T. J. Sensitivity analysis for unmeasured confounding in meta-analyses. J. Am. Stat. Assoc. 115, 163-172 (2020).

8. Damkier, P., Pottegård, A., dePont Christensen, R. \& Hallas, J. Annotations and reflections: pregnancy and paracetamol: methodological considerations on the study of associations between in utero exposure to drugs and childhood neurodevelopment. Basic Clin Pharmacol. Toxicol. 116, 2-5 (2015).

9. Eichler, J. et al. Maternal depressive symptoms and stress during pregnancy as predictors of gestational age at birth and standardized body mass index from birth up to 2 years of age. BMC Pregnancy Childbirth 21, 635 (2021).

10. Labor, S. et al. What is safe enough - asthma in pregnancy - a review of current literature and recommendations. Asthma Res. Pract. 4, 11 (2018).

Competing interests

A.R.S. declares past consulting for Cadence Pharmaceuticals. The other authors declare no competing interests.

\section{Supplementary information}

The online version contains supplementary material available at https://doi.org/10.1038/s41574-021-00606-x. 